\title{
Direct observation of a surface resonance state and surface band inversion control in black phosphorus
}

\author{
N. Ehlen, ${ }^{1, *}$ A. Sanna, ${ }^{2}$ B. V. Senkovskiy, ${ }^{1}$ L. Petaccia,${ }^{3}$ A. V. Fedorov,${ }^{1,4}$ G. Profeta, ${ }^{5}$ and A. Grüneis ${ }^{1, \dagger}$ \\ ${ }^{1}$ II. Physikalisches Institut, Universität zu Köln, Zülpicher Strasse 77, D-50937 Köln, Germany \\ ${ }^{2}$ Max-Planck-Institut für Mikrostrukturphysik, Weinberg 2, D-06120 Halle, Germany \\ ${ }^{3}$ Elettra Sincrotrone Trieste, Strada Statale 14 km 163.5, I-34149 Trieste, Italy \\ ${ }^{4}$ IFW Dresden, P.O. Box 270116, Dresden D-01171, Germany \\ ${ }^{5}$ Department of Physical and Chemical Sciences and SPIN-CNR, University of L'Aquila, Via Vetoio 10, I-67100 Coppito, Italy
}

(Received 19 November 2017; published 24 January 2018)

\begin{abstract}
We report a Cs-doping-induced band inversion and the direct observation of a surface resonance state with an elliptical Fermi surface in black phosphorus (BP) using angle-resolved photoemission spectroscopy. By selectively inducing a higher electron concentration $\left(1.7 \times 10^{14} \mathrm{~cm}^{-2}\right)$ in the topmost layer, the changes in the Coulomb potential are sufficiently large to cause surface band inversion between the parabolic valence band of BP and a parabolic surface state around the $\Gamma$ point of the BP Brillouin zone. Tight-binding calculations reveal that band gap openings at the crossing points in the two high-symmetry directions of the Brillouin zone require out-of-plane hopping and breaking of the glide mirror symmetry. Ab initio calculations are in very good agreement with the experiment if a stacking fault on the BP surface is taken into account. The demonstrated level of control over the band structure suggests the potential application of few-layer phosphorene in topological field-effect transistors.
\end{abstract}

DOI: 10.1103/PhysRevB.97.045143

\section{INTRODUCTION}

The recent synthesis of metallic black phosphorus (BP) $[1,2]$, the prediction of electron-phonon-mediated superconductivity in lithium-doped BP [2], and its experimental confirmation [3] with $T_{C}=3.8 \mathrm{~K}$ have spurred additional interest in alkali-metal-doped BP as it represents an interesting playground to test fundamental physics and technologically relevant effects. In particular, the control of the charge carrier concentration and the electron energy band gap of BP is fundamentally important. The high electron mobility in BP allowed for the fabrication of field-effect transistors (FETs) [4-9] and photodetectors [10] with good characteristics. An insulator-tometal transition has also been achieved by ionic liquid gating [11]. It has been shown that potassium adsorption onto the BP surface closes the band gap, and an anisotropic Dirac cone was revealed by angle-resolved photoemission spectroscopy (ARPES) [1]. A gate-tunable Stark effect, i.e., the electronic control of the band gap by electric field, has been demonstrated and is directly relevant for optoelectronic applications of BP [12]. An in situ electric transport study of the conductivity of the surface of BP showed a huge increase in the conductance and a reduction of the gap upon potassium deposition [13]. This work also hinted at the existence of surface resonant states [13]. The underlying mechanism for understanding these experiments is a charge accumulation on the surface of BP or few-layer phosphorene which can be controlled by an external gate voltage. The physics of surface charge doping in BP must also consider the existence of a surface resonance state. Surface

\footnotetext{
*ehlen@ph2.uni-koeln.de

†'grueneis@ph2.uni-koeln.de
}

resonance states have been reported using ARPES [14,15], but a complete ARPES characterization of their dispersion relation is missing. A surface resonance state is localized on the surface of BP, but its energy can lie outside the band gap [16], allowing hybridization with bulk states. Surface states and surface resonance states also play a decisive role for applications of BP beyond standard field-effect control of the carrier concentration. For example, it has been theoretically suggested that an electric field perpendicular to the layers is a means to invert the band gap and to engineer topologically protected edge states in few-layer phosphorene [17,18]. A band inversion of the parabolic valence and conduction bands would result in crossing points that lie along an ellipse in $k$ space. For cuts along the $\Gamma X$ and $\Gamma Y$ high-symmetry directions of the orthorhombic unit cell $[17,18]$, we would expect two crossing points each. Tight-binding (TB) calculations and a symmetry analysis suggest that the band crossing in the $\Gamma Y$ direction is protected by glide mirror symmetry $[19,20]$. Experimentally, ARPES measurements of K-doped BP showed a band inversion upon strong K doping [1], and a very recent work [21] analyzed the two high-symmetry crossing points in more detail and found that a gap opens in the $\Gamma X$ direction, while the size of the gap in $\Gamma Y$ is below the energy resolution. However, a quantitative description of the band inversion observed in ARPES including the effects of interlayer hopping and glide mirror symmetry breaking is still missing. Such realistic models are needed, for example, for the simulation of topological FETs.

This paper addresses these issues and unravels the nature of the surface resonance state and its connection with the increased conductivity and the topology of the band structure. We directly show, using ARPES, that Cs adsorption on the surface of BP induces a massive doping and leads to a surface 
band inversion between the valence band states of BP and a surface band. The energy of the band inversion observed here for $\mathrm{Cs}$ doping is larger than for $\mathrm{K}$ doping. We map the full three-dimensional surface-band-inverted Dirac cone and demonstrate that the conduction band is a surface resonance state localized on the topmost phosphorene layer, while the valence band is localized on the subsurface layers below. The region of the inverted bands is accurately mapped and clearly shows the overlapping surface and bulk states. A quantitative TB model developed for pristine BP [22] is adapted for doped BP. It demonstrates the requirements for protecting the band crossings imposed on crystal symmetry. These TB simulations also show that, upon populating the surface resonance state, the interlayer interaction decreases. A microscopic model is obtained from first-principles density functional theory (DFT) calculations. DFT suggests that Cs doping is accompanied by the formation of a stacking fault that decouples surface layers from the substrate. The ARPES spectrum and the occurrence of a band inversion with a negligible gap opening are accurately reproduced by DFT.

\section{ANGLE-RESOLVED PHOTOEMISSION SPECTROSCOPY}

ARPES was performed at the BaDElPh beamline [23] of the Elettra synchrotron in Trieste, Italy, with linear $s$ and $p$ polarization at temperatures of $20 \mathrm{~K}$. The crystal surfaces were prepared in situ in a vacuum better than $5 \times 10^{-11} \mathrm{mbar}$ and at liquid-He temperatures by cleaving with a top post inside the analysis chamber [22]. Immediately after the cleave, we determined the high-symmetry directions through lowenergy electron diffraction. Cs deposition was carried out in an ultrahigh-vacuum (UHV) chamber from SAES Getters with the sample at $20 \mathrm{~K}$. We performed stepwise evaporation of $\mathrm{Cs}$, which we monitored by ARPES measurements of the band structure. We found that the changes in the band structure with an increasing amount of Cs deposited are very similar to the ones already reported for $\mathrm{K}$ doping [1]. Cs evaporation was stopped after the maximum doping level was reached. Figures 1(a) and 1(b) show sketches of the functionalized BP surface and of the surface Brillouin zone of BP with $\Gamma, X$,
$Y$, and $S$ high-symmetry points. Prior to Cs deposition we recorded ARPES of the pristine BP crystal to confirm the surface quality and the orientation. We have obtained results in agreement with those published earlier [2,22]. Figures 1(c) and 1(d) show ARPES spectra (taken at $h v=21 \mathrm{eV}$ with a horizontal entrance slit on the analyzer) after maximum deposition of Cs. These spectra were recorded along the $\Gamma Y$ direction for $p$-polarized light and $s$-polarized light, respectively. In Fig. 1(c) we also show a fit of the parabolic band dispersion of the electronlike band which yields an effective electron mass of $m^{*}=1.38 m_{e}\left(m_{e}\right.$ is the free-electron mass). Figure 1(e) shows the resulting spectrum from adding Figs. 1(c) and 1(d). We find that $p$-polarized light couples mainly to the electronlike band, while $s$-polarized light couples to the holelike band. The photoemission intensities for $s$ - and $p$ - polarized light also suggest that the electron- and holelike bands have close to orthogonal symmetries. The ARPES spectra feature a sharp electronlike band and a slightly blurry holelike band. As we will show by DFT, this difference is related to the nature of the wave function of the electronlike band which is localized on the topmost layer and of the holelike band which, on the contrary, is delocalized over several layers underneath the surface. It can be seen that the surface conduction band minimum in Fig. 1(c) is lower in energy than the valence band maximum in Fig. 1(d), indicating a band inversion at the surface.

The dispersion of the surface resonance state and the holelike valence band is studied in more detail by performing an ARPES mapping in the full two-dimensional (2D) Brillouin zone (BZ). Figure 2 displays the full three-dimensional experimental ARPES intensity maxima. Maxima of equienergy contours were obtained from fitting peak ARPES intensities in the radial cuts centered around $\Gamma$. The elliptical shape of the equienergy contours can clearly be seen. The ellipses have their short (long) axis in the $\Gamma X(\Gamma Y)$ directions. The observed anisotropy is consistent with previous optical [24] and scanning tunneling microscopy measurements [25] of pristine BP and supports the band assignment to a phosphorene-derived band. This anisotropy is rooted in the puckered structure of BP and results in a quadratic energy dispersion along $\Gamma Y$ and a linear energy dispersion along $\Gamma X[22]$. The ARPES intensity has an inverse angular distribution for energy contours below and
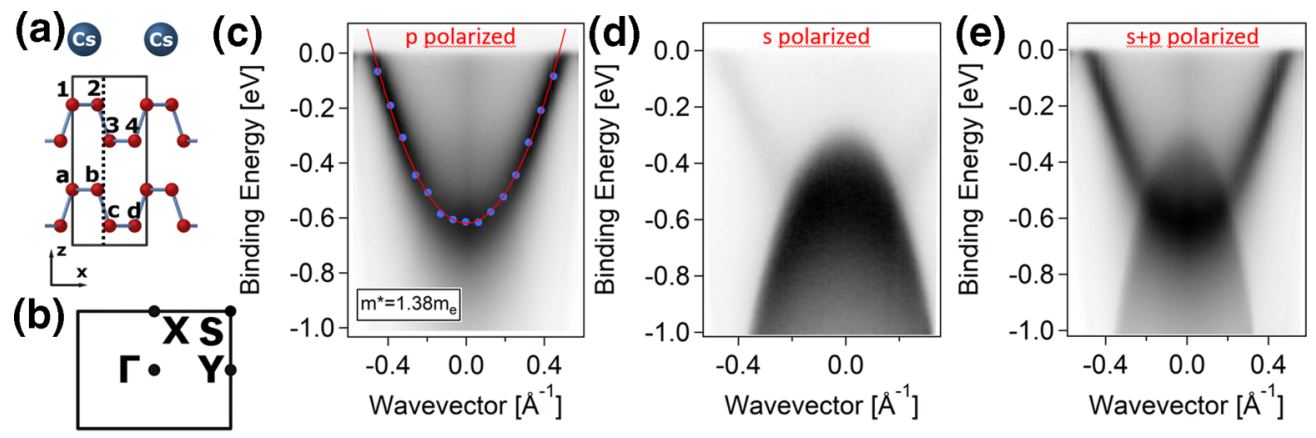

FIG. 1. (a) Sketch of the bilayer phosphorene structure with Cs adsorbed. The dashed line denotes the mirror plane of the glide mirror symmetry. The glide axes are in the $x$ and $y$ directions. The four atoms in the bilayer unit cell are indicated by $a-d$ and $1-4$. (b) The surface $\mathrm{BZ}$ of BP and the high-symmetry points. Two-dimensional, $k$-dependent ARPES spectra along the $\Gamma Y$ direction measured with (c) $p$ - and (d) $s$-polarized light. A fit of the effective electron mass yielding $m^{*}=1.38 m_{e}$ is shown $\left(m_{e}\right.$ is the free-electron mass). (e) The $s$ - and $p$-polarized spectra added together. 


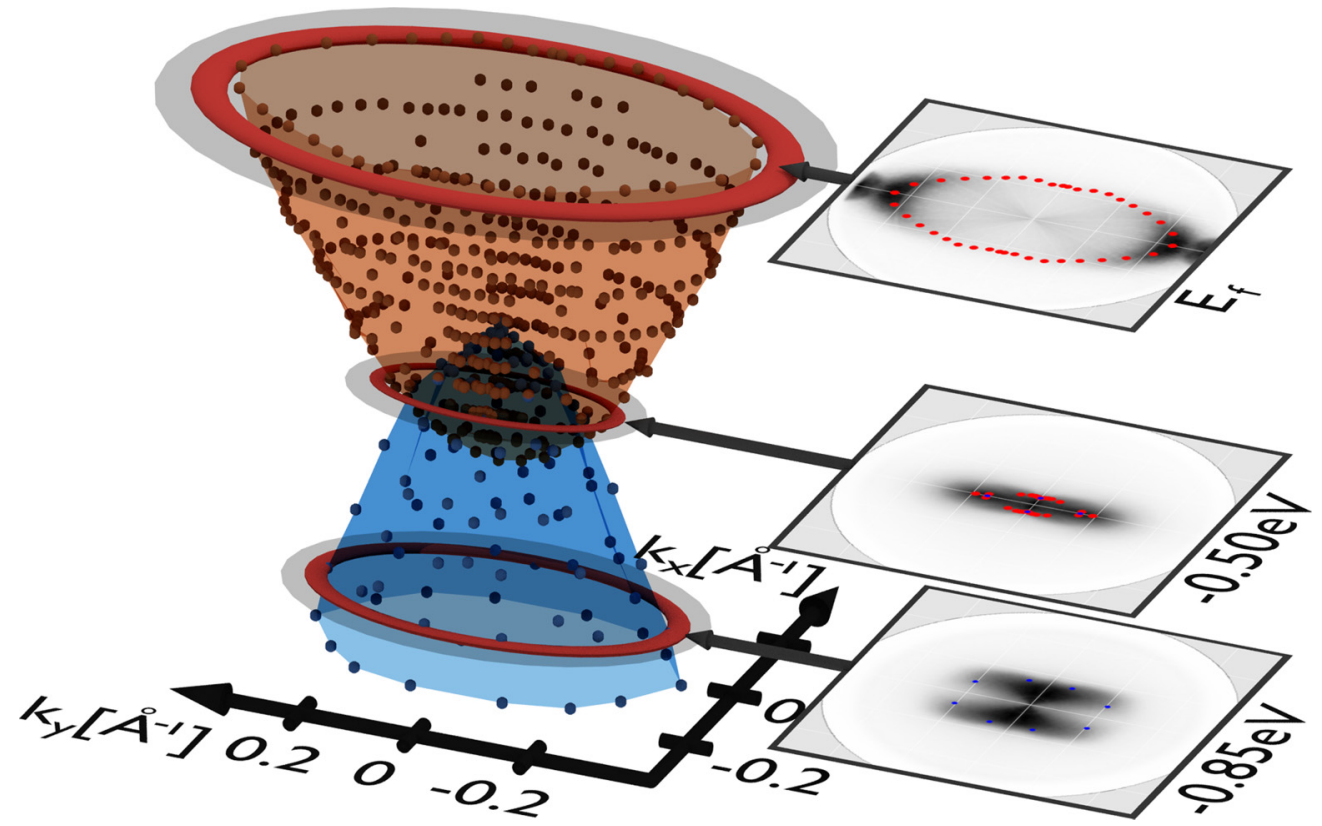

FIG. 2. Three-dimensional energy band dispersion of BP with a monolayer of Cs adsorbed on its surface. The points are the experimental ARPES maxima (measured at $h v=21 \mathrm{eV}$ with $s$ - and $p$-polarized light), and the surfaces are an interpolation of the symmetrized data. Red (blue) denotes electronlike (holelike) bands. The right part shows equienergy contours of ARPES data for three binding energies. Additional equienergy contours (see the Appendix) were used to interpolate the three-dimensional dispersion. The left part originates from radial fits to the surfaces of the kind shown on the right.

above the region of band inversion because these states have a different character and hence matrix element. Nevertheless, it is possible to reliably extract ARPES maxima, and more equienergy contours are shown in the Appendix. From ARPES equienergy contours all relevant parameters describing the surface band can be extracted. The region of surface band inversion is clearly seen around the $\Gamma$ point. Fermi velocities of $3.68 \times 10^{5}$ and $1.02 \times 10^{6} \mathrm{~m} / \mathrm{s}$ are found in the $\Gamma Y$ and $\Gamma X$ directions, respectively. We find a charge carrier concentration of $1.7 \times 10^{14} \mathrm{~cm}^{-2}$. The Fermi velocities are in a range similar to those reported previously for electron-doped BP [1]. The differences are ascribed to the larger doping level we have here.

\section{X-RAY PHOTOEMISSION SPECTROSCOPY AND NEAR-EDGE X-RAY ABSORPTION FINE STRUCTURE}

The localization on the BP surface is also consistent with the fact that monolayer Cs does not intercalate into the bulk of $\mathrm{BP}$. This is evident from angle-dependent $\mathrm{x}$-ray photoemission spectroscopy (XPS). XPS measurements have been performed at the German-Russian Beamline (RGBL) at the BESSY II synchrotron in Berlin using a photon energy $h v=300 \mathrm{eV}$. The results can be seen in Figs. 3(a) and 3(b), which show the XPS spectrum for grazing and normal emission on Cs-doped BP. The spectra are normalized to the $\mathrm{P} 2 p$ core-level intensity. It can be seen that upon doping, the P $2 p$ core levels shift
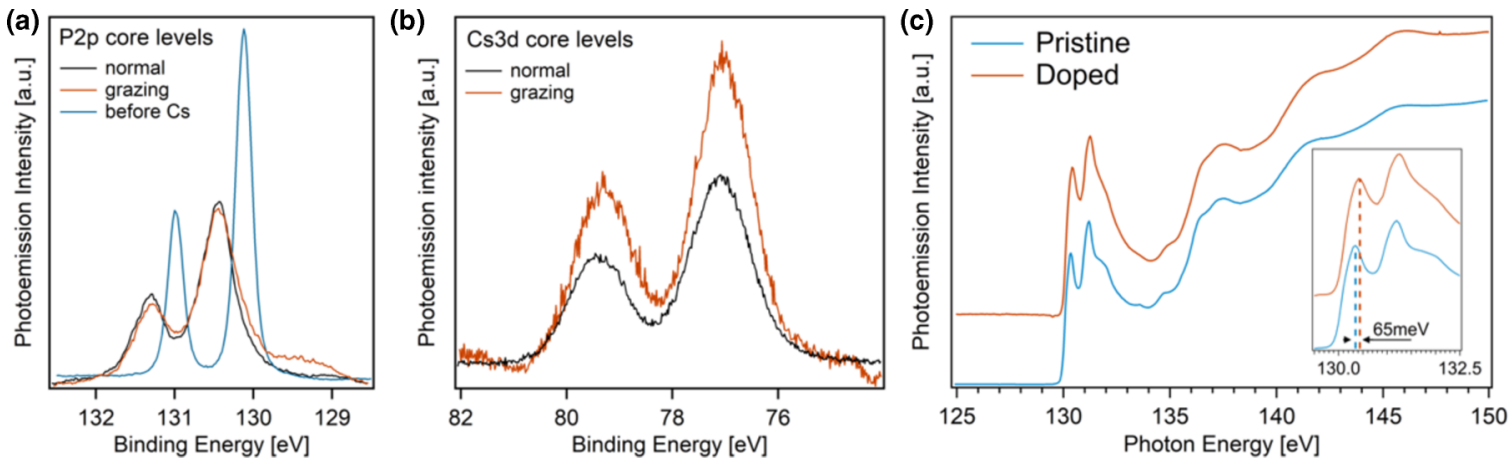

FIG. 3. (a) XPS spectra $(h v=300 \mathrm{eV})$ of the P $2 p$ core levels before doping (blue) and after Cs doping (black and red). The black spectrum is for normal emission, and the red spectrum is for grazing emission. (b) Cs $3 d$ core-level spectrum ( $h v=300 \mathrm{eV}$ ) for normal emission (black) and for grazing emission (red). These two spectra are normalized to the corresponding P $2 p$ core-level spectra shown in (a). (c) Near-edge x-ray absorption fine-structure spectrum of pristine (blue) and Cs-doped (red) BP measured in the total-electron-yield configuration. The inset shows that the onset of the absorption edge for doped BP is upshifted by $\sim 65 \mathrm{meV}$ when compared to pristine BP. 
down to higher binding energy, indicating a charge transfer from $\mathrm{Cs}$ to $\mathrm{P}$. The Cs $3 d$ core levels show an increase in intensity under grazing emission when compared to $\mathrm{P} 2 p$ core levels measured under identical emission angles. This is a clear indicator that Cs lies preferentially on top of the BP surface. Regarding additional evidence of the nature of the surface state, we performed measurements of the near-edge $\mathrm{x}$-ray absorption fine structure (NEXAFS) and observed an upshift of $\sim 65 \mathrm{meV}$ in the phosphorene $2 p$ absorption edge. Figure 3(c) shows the relevant spectra. The observed shift could be related to the filling of the empty (surface) states upon Cs doping. Due to Pauli blocking, the energetically lowest lying transitions in NEXAFS become forbidden. This in turn shifts the $\mathrm{P}$ absorption edge to higher values. This indicates that this band has partial $\mathrm{P}$ character.

\section{TIGHT-BINDING DESCRIPTION}

\section{A. Interlayer interaction and glide mirror symmetry}

For further analysis shown in Fig. 4, we consider the sum of $s$ - and $p$-polarized ARPES spectra outlined in the previous section. Figure 4(a) shows the sum of the individual spectra together with a TB calculation along the $\Gamma Y$ direction. Figure 4(b) depicts an energy distribution curve from Fig. 4(a) at $k_{Y}=0$ with two maxima as indicated, corresponding to the intensity maxima of electron- and holelike bands. Figures 4(c) and 4(d) depict the raw ARPES data and their second derivative along the $\Gamma X$ crystallographic direction (the second derivative is shown to enhance the contrast of the features) together with results of the TB calculation. While the bands are parabolic along $\Gamma Y$, the situation is quite different along the $\Gamma X$

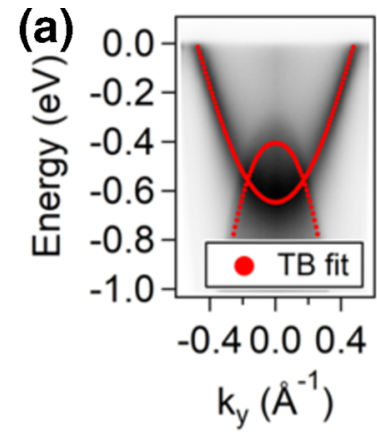

(e)

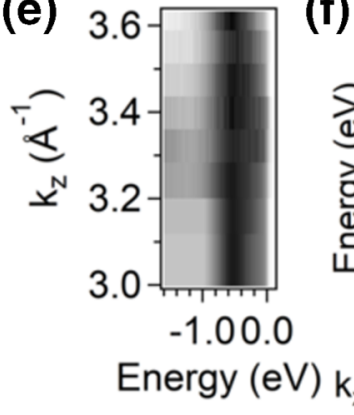

(f)

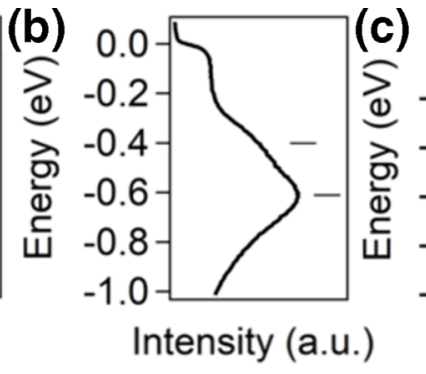

(g)
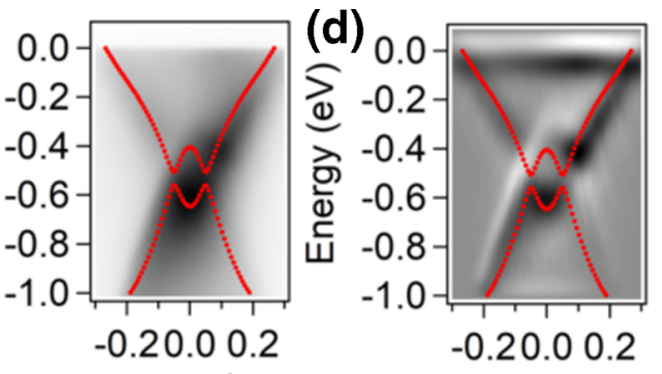

$\mathrm{k}_{\mathrm{x}}\left(\AA^{-1}\right)$
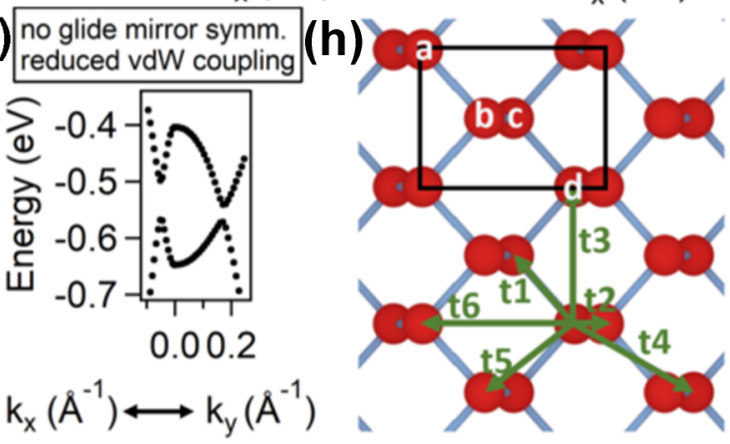

(j)

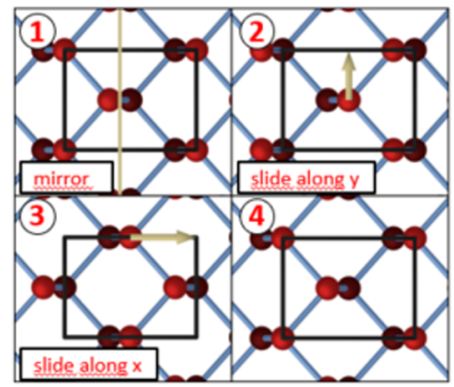

(k)

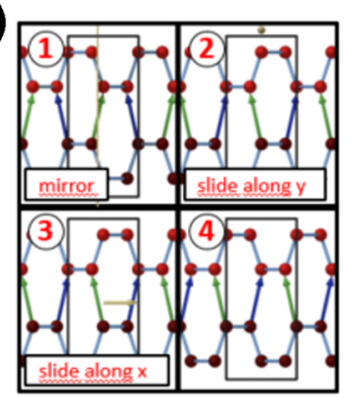

FIG. 4. (a) ARPES scans along the $\Gamma Y$ direction along with a tight-binding (TB) fit. (b) Energy distribution curve at $k=0$ indicating two peaks at $E=-0.4 \mathrm{eV}$ and $E=-0.6 \mathrm{eV}$. (c) ARPES scan along $\Gamma X$ with a TB fit. (d) Second derivative of the $\Gamma X$ scan overlaid on TB results. (e) $k_{z}$ scan using a photon energy sweep between 15 and $40 \mathrm{eV}$ and the inner potential given in Ref. [22]. (f) and (g) TB calculations assuming an out-of-plane coupling of bulk BP and a TB parametrization that breaks the glide mirror symmetry, respectively. (h) In-plane TB parameters up to the sixth nearest neighbor. (i) Nearest and next-nearest out-of-plane TB parameters. (j) Glide mirror symmetry operations for monolayer phosphorene. (k) Glide mirror symmetry operations for bilayer phosphorene. The arrows denote the nearest-neighbor out-of-plane hopping (parameter $s$ ). The Supplemental Material [26] contains a video animation of the glide mirror symmetry operations. 
crystallographic direction: here the bands are almost linear. Photon-energy-dependent ARPES allows us to gain information on the spatial localization of the newly found band. The results are shown in Fig. 4(e), indicating a completely flat $k_{z}$ dispersion as a function of the energy (wave vector perpendicular to the surface) and thus a well-localized surface state. Note that bulk valence bands have been measured under identical conditions and were found to disperse with photon energy [22]. From the TB fit of Fig. 4(a) we directly observe a band inversion of hole- and electronlike bands close to the $\Gamma$ point along the $\Gamma Y$ crystallographic direction at $k_{Y}= \pm 0.17 \AA^{-1}$. The points where hole- and electronlike bands almost touch each other in the $\Gamma X$ direction appear at $k_{X}= \pm 0.06 \AA^{-1}$ [see TB fits in Figs. 4(c) and 4(d)]. If we use the out-of-plane TB parameters which accurately describe the bulk [22], the band opening along $\Gamma X$ is a factor of 5 larger than the experimentally observed overlap between electron- and holelike bands. This is shown in Fig. 4(f), and it is clear that it does not agree with the experimental ARPES results along $\Gamma X$. To correctly describe ARPES data along $\Gamma X$, we resort to out-of-plane hopping parameters smaller than those for bulk BP. Reducing the parameters by one order of magnitude, we obtain results in excellent agreement with the observed ARPES spectra. The TB calculations along $\Gamma X$ shown in Fig. 4(c) have been performed with these parameters. The strong decrease in out-of-plane hopping is a hint towards a decrease in the interlayer interaction and will be addressed in more detail by ab initio calculations shown later. Finally, let us explore the effects of glide mirror symmetry breaking. This is linked to the band crossing along $\Gamma Y$, which is stable against any choice of parameters unless glide mirror symmetry is broken. We can address this issue, using TB, purposefully, e.g., by using a different coupling between symmetrically located atoms in the upper and lower layers. We break the glide mirror symmetry of the Hamiltonian by introducing different values for the next-nearest out-ofplane hopping parameters of symmetrically located hopping directions. In the example calculation shown in Fig. 4(g) we assumed a factor of 2 difference in the nearest-neighbor out-of-plane hopping and kept all other parameters equal to the TB parameters used to calculate the bands in Figs. 4(a), 4(c) and 4(d). Our calculations indicate that, indeed, a band gap in the $\Gamma Y$ direction is opened if we break glide mirror symmetry in this way. A video animation in the Supplemental Material illustrates that glide mirror symmetry breaking results in different TB values for the $s 1$ parameter connecting atoms $b-3$ and $a-4$ [26]. Experimentally, it is not clear by how much the randomly adsorbed Cs breaks the glide mirror symmetry. Figure $4(\mathrm{~g})$ thus depicts a general case of the band structure of bilayer phosphorene affected by both interlayer coupling and glide mirror symmetry breaking. This is useful not only for describing adsorption but also for describing substrate interaction in future works. The set of TB parameters which was used to calculate Fig. 4(g) is given in the Appendix. The calculations in Figs. 4(a), 4(c) and 4(d) can be reproduced with this set of TB parameters, too. The glide mirror symmetry can be restored by using only a single next-nearest-neighbor hopping as described in the Appendix.

\section{B. Tight-binding Hamiltonian}

Let us now discuss the details of the TB calculations. We have used an effective bilayer model with a different on-site potential on each layer. This method has been successfully applied previously and does not require us to account for the puckered nature of the layers (i.e., a different on-site potential within one phosphorene layer) [27,28]. The choice of different on-site potentials describes the effects of gating without taking into account the microscopic nature of the $\mathrm{Cs}$ adsorption. For a given in-plane Hamiltonian $H_{0}$ from Ref. [22] we set up a bilayer Hamiltonian by creating a block-diagonal Hamiltonian matrix with the original $H_{0}$ on the block diagonal. The on-site potentials can then be modeled by adding matrices with parameters $\Delta_{1}$ and $\Delta_{2}$ along their diagonal (and zero elsewhere) to $H_{0}$. This lifts the degeneracy of the bands. The meaning of such a staggered potential is to account for a higher electron density on the topmost layer as it is in close vicinity to the positively charged Cs ions. Thus the electron energy band mainly located on the topmost layer will be downshifted with respect to the band located on the subsurface layers farther away from Cs. The interlayer interaction is introduced by an out-of-plane hopping Hamiltonian $H_{\text {oop }}$ [22] in the off-diagonal blocks. The full Hamiltonian then becomes

$$
H=\left[\begin{array}{cc}
H_{0}+\Delta_{1} \cdot 11 & H_{o o p} \\
H_{o o p}^{*} & H_{0}+\Delta_{2} \cdot 11
\end{array}\right] .
$$

The hopping parameters for the in-plane Hamiltonian $H_{0}$ and the out-of-plane Hamiltonian $H_{\text {oop }}$ are illustrated in Figs. 1(h) and 1(i). The band inversion is a result of parameters $\Delta_{1}$ and $\Delta_{2}$, which cause the shifts of valence and conduction bands.

\section{Graphical representation of glide mirror symmetry}

Figure 4(j) illustrates the glide mirror symmetry operations for monolayer phosphorene which involve one mirror operation and two translations by half a lattice vector along $x$ and $y$, respectively. It can be seen that after application of these three operations, the original configuration is recovered. The same procedure can be applied for bilayer phosphorene, which is the basis for our model. Figure 4(k) depicts how the lattice of bilayer phosphorene changes upon application of each of the three symmetry operations and that it eventually goes back to the original lattice. In Fig. 4(k) we also illustrate that the equality of the nearest out-of-plane hopping [parameter $s 1$ in Fig. 4(i)] between atoms $b-3$ and $a-4$ is protected by glide mirror symmetry. It can directly be seen that different hopping parameters between these atoms describe the violation of glide mirror symmetry. The TB calculations of Fig. 4(f) use equal values for the hopping $s 1$ between atoms $b-3$ and $a-4$, and no gap opens along $\Gamma Y$. On the other hand, the TB calculations of Fig. 4(g) use different hopping values of $s 1$ between these two pairs of atoms. As a consequence of this symmetry breaking, a gap is opened in the $\Gamma Y$ direction.

\section{AB INITIO CALCULATIONS}

As the TB includes the effects of the Cs atoms only indirectly (via the electrostatic doping with a staggered 
potential and symmetry breaking as discussed previously), it is interesting to build a microscopic model of Cs adsorbed on the BP surface. Here we perform $a b$ initio calculations to calculate the ground-state geometry of Cs on the BP surface and the relative band structure. Since it is impossible to model a random distribution of Cs atoms on the surface, we settled on modeling an ordered structure of $\mathrm{Cs}$ atoms at the surface that reproduce the observed doping. The exact location of the Cs atoms is obtained by structural relaxation. The ordered nature of the simulated Cs doping might induce some extra symmetry breaking that may not occur in the real system with a random Cs distribution. We found that the simulations still correctly describe the effect of charge transfer from Cs to P layers and doping, which is the most important effect of the Cs deposition. We have performed an extensive $a b$ initio characterization by modeling the system as a two-layer slab of BP with Cs adsorbed on one surface at the lowest-energy position obtained by energy minimization. Before settling on the description via bilayer systems, we checked several slab thicknesses with $a b$ initio calculations (one of which is shown in the Appendix). However, for the relevant results (localization of the bands in the presence of stacking faults) the extra layers have negligible effects, while the added bands complicate the band structures. The resulting band structures are reported in Fig. 5 for different stackings and dopings. In Fig. 5(a) we show the band structure for a two-layer slab with one Cs atom per surface unit cell. Although band inversion at the $\Gamma$ point is nicely reproduced by our calculation, the experimentally observed band crossing along the $\Gamma X$ and $\Gamma Y$ directions is not perfectly reproduced at any simulated doping level. In fact, a large anticrossing is always observed in the $\Gamma X$ direction, and a smaller one is observed in the $\Gamma Y$ direction. This also occurs at any slab thickness (up to six-monolayer slabs considered in our simulations). The character of the two anticrossing bands in Fig. 5(a) is clearly seen by projecting selected (arrows in Fig. 5) Kohn-Sham wave functions $\phi_{n k}(\mathbf{r})$ with band index $n$ at wave vector $k$ on the phosphorus-centered atomic orbitals. The integrated wave function of the electronlike surface state indicates that this state is mostly localized on the topmost $\mathrm{P}$ layer with a less than $15 \%$ contribution from Cs. We can also gain information on the out-of-plane $(z)$ profile of the projection [see inset in Fig. 5(a)]. The inverted electronlike band [indicated by an orange arrow in Fig. 5(a)] is a surface state, located on the topmost layer, with a small component located on Cs. The holelike band is located on the second layer of the bilayer simulation. However, for real systems with several layers, the holelike state extends into the bulk (see the Appendix). This nicely explains the sharper ARPES features
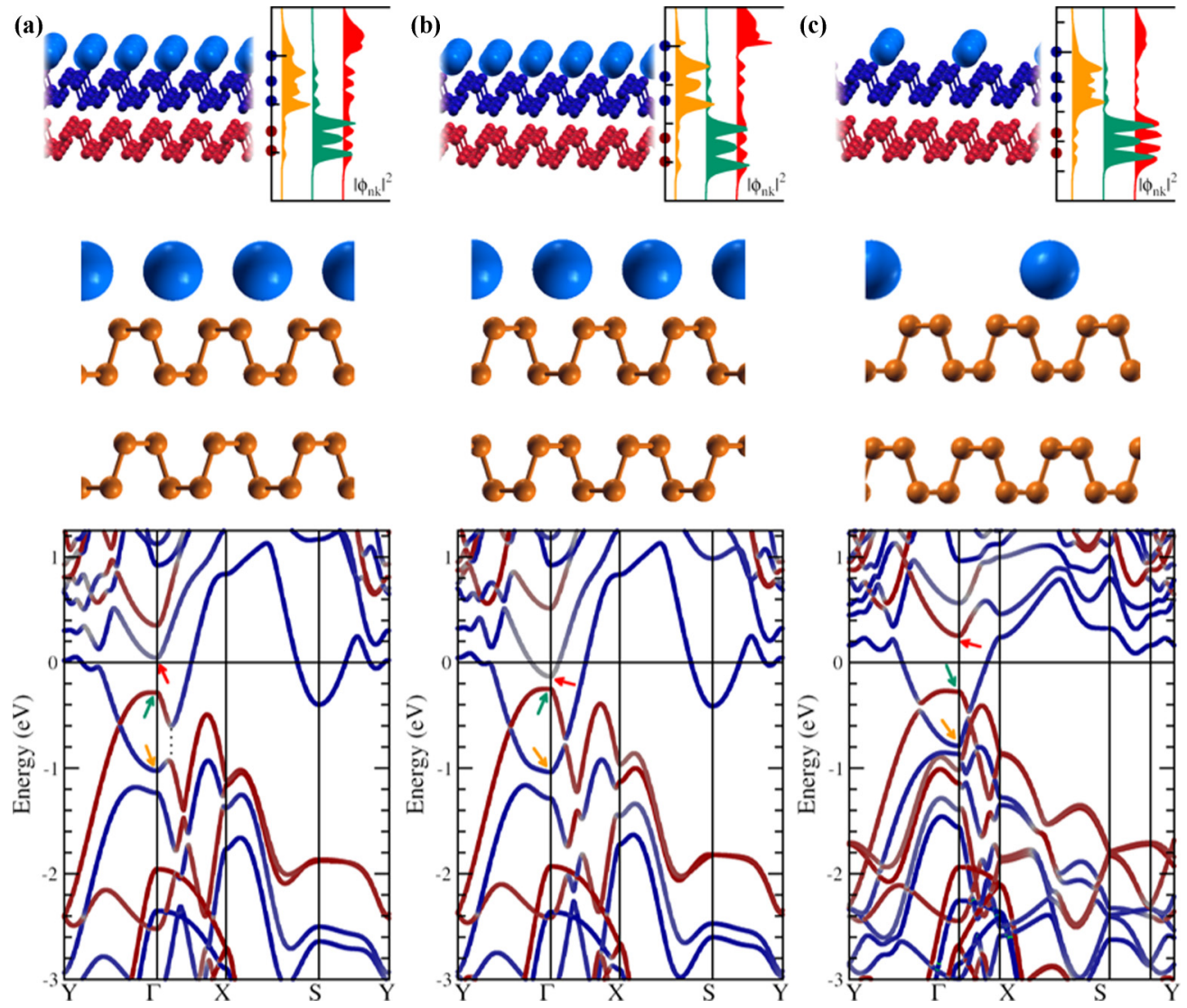

FIG. 5. Structure and electron energy bands of Cs-doped bilayer phosphorene. (a) One Cs atom per unit cell. (b) One Cs atom per unit cell with inverse stacking. (c) One Cs atom per two unit cells with inverse stacking. Colors in the band plots give the projection onto the top layer, Cs (blue), and the bottom layer (dark red). For some selected states at $\Gamma$ (colored arrows) we show the $|\phi|^{2}$ integrated in plane and as a function of the out-of-plane coordinate $z$. 
for the electronlike band, which is localized only on the top layer, as opposed to the holelike band, which is delocalized over several layers, resulting in broader emission spectra. Since the electronlike band overlaps with the bulklike hole states, it is a surface resonance state. In spite of the overall different orbital character, the tails of these wave functions clearly overlap significantly, allowing a gap opening of $\sim 400 \mathrm{meV}$ at the crossing energies, which exceeds the experimental upper bound of $200 \mathrm{meV}$. It is evident that a refinement of the model is required in order to reproduce the observed experimental band dispersion. We find that this discrepancy can be neatly explained if we consider the possibility of a stacking fault. While the BP stacking is energetically the most favorable (we verified this even in the presence of Cs), the energy differences between different stacking orders of the phosphorene layers are extremely small (of the order of $\sim 1.5 \mathrm{meV} /$ atom), and many effects could alter the stacking, including impurities on the BP substrate or adsorbed Cs. Notably, a change in stacking order is a common situation in van der Waals layered materials, for example, in graphite intercalation compounds, where the typical $\mathrm{AB}$ (or Bernal) stacking reverts to an $\mathrm{AA}$ stacking upon intercalation of alkali atoms [29]. We consider a reverse stacking in which the topmost phosphorene layer is shifted along the armchair direction. The new crystal structure and the calculated band structure are shown in Fig. 5(b). This effect clearly reduces significantly the interaction between hole and electron bands and leads to a band inversion qualitatively identical to the ARPES measurements. This indicates that Cs doping can induce a modification on the BP surface, electronically detaching it from the substrate. For the unit cell shown in Fig. 5(c) the position of the Fermi level is in excellent agreement with the experiment. The band structure is also similar to the experimental one as the extra states around the $S$ point have moved above the Fermi level. Note there is a band folding due to the larger supercell used here, so extra bands appear in the $\Gamma X$ direction close to the crossing point. The new Cs positions break the glide mirror symmetry that protects the band crossing; this is why the band gap in Fig. 5(c) is opened along the $\Gamma Y$ direction.

\section{CONCLUSIONS AND OUTLOOK}

We have shown that the adsorption of a Cs monolayer induces a surface resonance state in BP with an elliptically shaped Fermi surface and an electron density of $1.7 \times$ $10^{14} \mathrm{~cm}^{-2}$. The energy-level structure is such that holelike and electronlike bands reverse their order around the $\Gamma$ point of the BZ. TB modeling of the ARPES data indicates a decrease in the interlayer interaction and shows directly that the potential of a random Cs distribution and nonzero interlayer interaction will open band gaps in the $\Gamma X$ and $\Gamma Y$ crystallographic directions. $A b$ initio calculations are in excellent agreement with the experiment if a stacking fault of the topmost layer by a shift along the armchair direction is taken into account. In this situation, the interlayer interaction decreases. These calculations also indicate that the wave functions of the electronlike and holelike bands are located on the topmost and subsurface phosphorene layers, respectively. The discovery of a surface resonance state poses the question of whether the observed surface resonance actually hybridizes with the bulk bands or whether the system can simply be thought of as a noninteracting superposition of the surface resonance band with the bulk band. In the latter case none of the suggested topological effects would come into play. Unfortunately, the energy resolution of ARPES is too low to clearly resolve the size of the gap. Thus we have to rely on the calculations discussed above. The theoretical results indicate the hybridization of the surface state with the bulk bands and show the gap formation at the crossing points, suggesting that the physics of bulk band inversion in BP might still be applicable here. Increasing interlayer distance that reduces the out-of-plane coupling might reduce the gap size but would not fundamentally alter the existence of the gap. This poses the question of whether the gap size is sufficiently large to be physically and technologically relevant. On the edge of a phosphorene flake a spin-polarized surface state is predicted to form inside the bulk gap [17]. Thus the operation temperature of a topological FET must be such that temperature cannot excite electrons between the bulk states. The discovery of a surface band inversion that is purely induced by a layer-dependent Coulomb potential [the staggered electric field introduced in Eq. (1)] should stimulate attempts to electronically control the surface band inversion, i.e., to build a switch. For a topological FET device, one wishes to have the crossing points at the Fermi level. This might be achieved in a dual-gate geometry [17] using field-effect or ionic liquid gating. Devices based on field-effect control of the band inversion are limited by the carrier density which can be induced by field-effect gating. The carrier density $n$ is limited by the capacitance $\epsilon$ and the dielectric strength of the gate oxide, which limits the gate voltage. We can induce a carrier density $n=\epsilon V / e$ (here $e$ is the elementary charge, and $V$ is the gate voltage). For $\epsilon \sim 20 \mathrm{nF} / \mathrm{cm}^{2}$ and $V=80$ $\mathrm{V}$ we reach $n=10^{13} \mathrm{~cm}^{-2}$. These values are indeed possible in experiments of standard FETs [7]. In the present work, we have achieved surface band inversion of about $300 \mathrm{meV}$ separation between valence band maximum and conduction band minimum at about the tenfold carrier density. Thus we expect that surface band inversion can already be achieved at carrier concentrations lower than $n=10^{14} \mathrm{~cm}^{-2}$. Nevertheless, to fabricate topological FETs high $\epsilon$ gate dielectrica might be needed.

Let us now discuss the effects of relative shifts in the energy location of the band gaps along the $\Gamma Y$ and $\Gamma X$ directions. The existence of a "clean gap" in both directions is necessary for the potential application of phosphorene in a topological FET. It should be noted that in the DFT calculations for Fig. 5(c) the band gaps visible along $\Gamma X$ and $\Gamma Y$ are located at different energies and do not overlap. In this case it would not be possible to build a topological FET from a slab of multilayer phosphorene. However, while a similar behavior is visible in the TB calculations, the band gap along $\Gamma Y$ is inside the band gap along $\Gamma X$ in that case. This discrepancy can be explained by the fact that small changes in the band structure can shift these band gaps relative to each other. We thus deem 
the theoretical results inconclusive on the nature of the gap. Transport measurements on bulk and few-layer flakes of the material should clarify this question.

Next, it would be interesting to extend the search to other alkali metals or alkali-earth metals [30,31]. In particular, $\mathrm{Li}$ doping from ARPES results seems to be much weaker compared to $\mathrm{K}$ and $\mathrm{Cs}$ doping; no surface state formation or band inversion is observed in Li-doped BP. With the knowledge of the strong size variation of the Fermi surfaces for these dopants, it is interesting to note the intercalant-independent critical temperature found in alkali-metal intercalated BP [3]. The discovery of superconductivity in alkali-metal intercalated $\mathrm{BP}$ raises the question of whether the surface resonance state sustains superconductivity. This would call for a combined UHV spectroscopy and transport experiment in which the surface functionalization and electrical conductivity can be determined in situ at low temperatures as a function of alkalimetal adsorption. In this context, it is interesting to note that the measured ARPES band structure did not exhibit "kinks" as a result of electron-phonon interaction as theoretically reported [2]. Such "kinks" were measured, e.g., for alkalimetal-doped, superconducting graphene [30]. The Eliashberg function of Li-doped BP suggests a strong coupling to P- and Li-derived phonon modes up to energies of $50 \mathrm{meV}$ [2]. In the present case of Cs-doped BP, we expect a large downshift of the dopant-derived phonons, rendering them unresolvable in ARPES. However, for the P-derived vibrations, no downshift is expected, and hence these vibrations could still be resolvable. Finally, the realization of a tunable 2D electron gas with different carrier densities is of fundamental interest for many applications, such as the direct coupling of surface plasmons to terahertz light, as exploited currently, e.g., in doped graphene layers [32]; thus the current work suggests the use of BP for terahertz applications.

\section{ACKNOWLEDGMENTS}

N.E., B.V.S., A.V.F., and A.G. acknowledge ERC Grant No. 648589 "SUPER-2D” and funding from DFG project CRC 1238 (project A1) and DFG project GR 3708/2-1. The research leading to these results has received funding from the European Community's Seventh Framework Programme (FP7/20072013) under Grant Agreement No. 312284 (CALIPSO). We thank Elettra for the allocation of synchrotron radiation beam time at the BaDElPh beamline. The stay at the Elettra synchrotron was supported by the CERIC-ERIC consortium. The authors thank HZB BESSY II for the beam time allocation.

\section{APPENDIX}

\section{Equienergy contours of the ARPES intensity}

The surface band structure was mapped by ARPES in the full 2D BZ. Here we show the fourfold symmetrized raw ARPES data from which we extracted the maxima to compose the inverted Dirac cone in Fig. 2. The ARPES maxima were evaluated by scanning the ARPES intensity in the radial direction. Figure 6 depicts eight individual equienergy contours in an energy range between the Fermi level and 950 meV binding energy.

\section{Tight-binding parameters}

Table I shows the TB parameters used for the TB calculations shown in Fig. 4(g) of the main text. The Hamiltonian for these calculations is described in Ref. [22]. The parameters

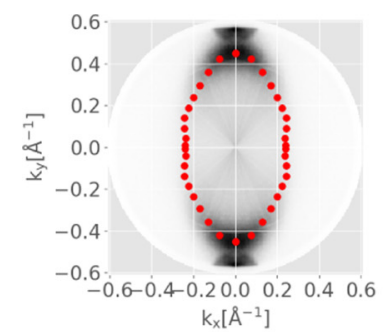

(a)

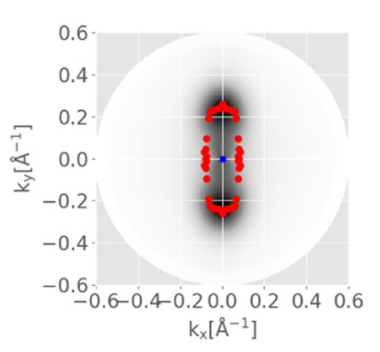

(e)

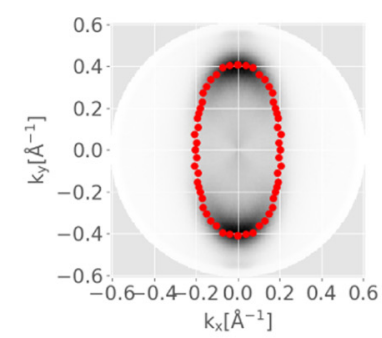

(b)

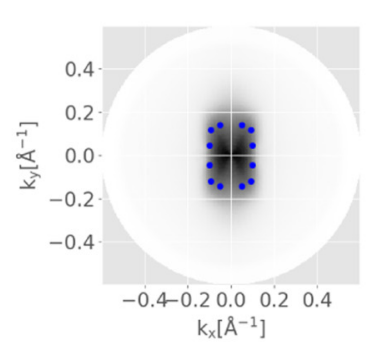

(f)

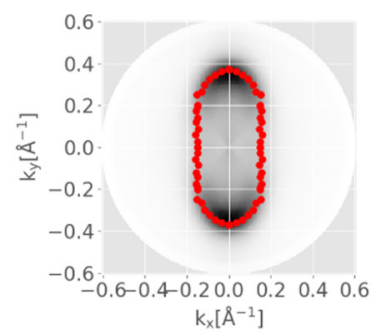

(c)

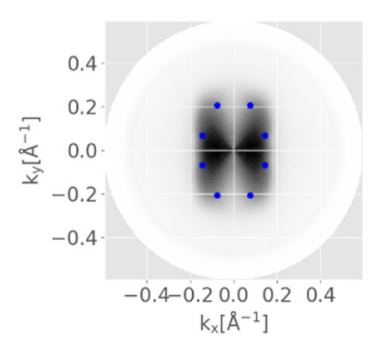

(g)

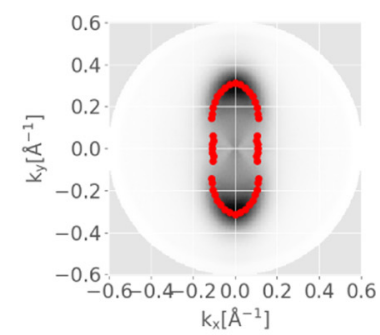

(d)

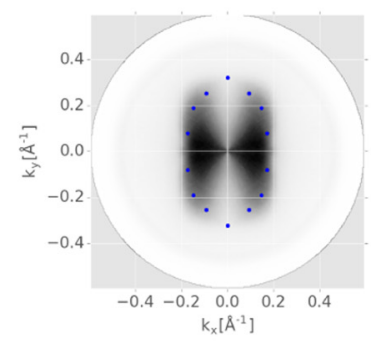

(h)

FIG. 6. Equienergy contours for (a) Fermi surface, (b) $100 \mathrm{meV}$, (c) $200 \mathrm{meV}$, (d) $300 \mathrm{meV}$, (e) $400 \mathrm{meV}$, (f) $700 \mathrm{meV}$, (g) $850 \mathrm{meV}$, and (h) $950 \mathrm{meV}$ binding energy. The red and blue dots are the maxima of the electronlike and holelike bands, respectively. 
TABLE I. The tight-binding parameters obtained from the fit to the ARPES data of Cs-doped BP. Figures 4(h) and 4(i) depict the hopping of $t 1-t 6$ and $s 1$ and $s 2$. By assuming different values for the first out-of-plane hopping parameter $(s 1)$ depending on the direction we artificially break the glide mirror symmetry that protects the band crossing. This is expressed by different hoppings between atoms $b-3$ and $a-4$. All values are in $\mathrm{eV}$.

\begin{tabular}{|c|c|c|c|c|c|}
\hline & Order & $V_{s s \sigma}$ & $V_{s p \sigma}$ & $V_{p p \sigma}$ & $V_{p p \pi}$ \\
\hline \multirow[t]{6}{*}{ In plane $(t 1-t 6)$} & $t 1$ & -4.4642 & 0.9836 & 4.1682 & -1.8475 \\
\hline & $t 2$ & -5.0011 & 3.9096 & 5.3775 & -0.7643 \\
\hline & $t 3$ & -1.1726 & 1.2455 & 1.6278 & 0.1603 \\
\hline & $t 4$ & -0.4074 & -0.0972 & 1.1678 & -0.3980 \\
\hline & $t 5$ & 1.0374 & 0.0672 & 0.8531 & -0.5446 \\
\hline & $t 6$ & 4.3436 & 1.6451 & 1.3796 & -0.4215 \\
\hline \multirow[t]{3}{*}{ Out of plane $(s 1-s 2)$} & $s 1(b-3)$ & 0.0170 & 0.1466 & 0.1382 & 0.0462 \\
\hline & $s 1(a-4)$ & 0.0085 & 0.0733 & 0.0691 & 0.0231 \\
\hline & $s 2$ & -0.0687 & 0.0496 & 0.0056 & -0.0222 \\
\hline \multirow[t]{2}{*}{ On site } & $\varepsilon_{s}$ & -14.9881 & & & \\
\hline & $\varepsilon_{p}$ & -1.2376 & & & \\
\hline \multirow[t]{2}{*}{ Potential } & $\Delta_{1}$ & -1.0867 & & & \\
\hline & $\Delta_{2}$ & -0.4199 & & & \\
\hline
\end{tabular}

in Table I purposefully break the mirror glide symmetry. This is achieved by assuming a factor of 2 higher values for the nearest-neighbor out-of-plane hopping between atoms $b$ - 3 than for atoms $a-4$. The TB calculations shown in Figs. 4(a), 4(c) and $4(d)$ can be obtained by setting the out-of-plane hopping for atoms $b-3$ equal to that for atoms $a-4$.

\section{Wave function localization for four-layer phosphorene}

In order to investigate the localization of the wave function for the electronlike and holelike bands, we performed $a b$ initio calculations of the band structure for Cs monolayer adsorbed onto a four-layer phosphorene. The resulting band structure is reported in Fig. 7. The main point is that in this conventional geometry the only surface states are those of the electronlike bands with important contributions from the Cs atoms (green and blue lines in Fig. 7), while all the hole states are delocalized in the slab. This is clearly seen by observing the plots of the in-plane integrals of the Kohn-Sham orbitals $\left|\phi_{n \mathbf{k}}\right|^{2}$.
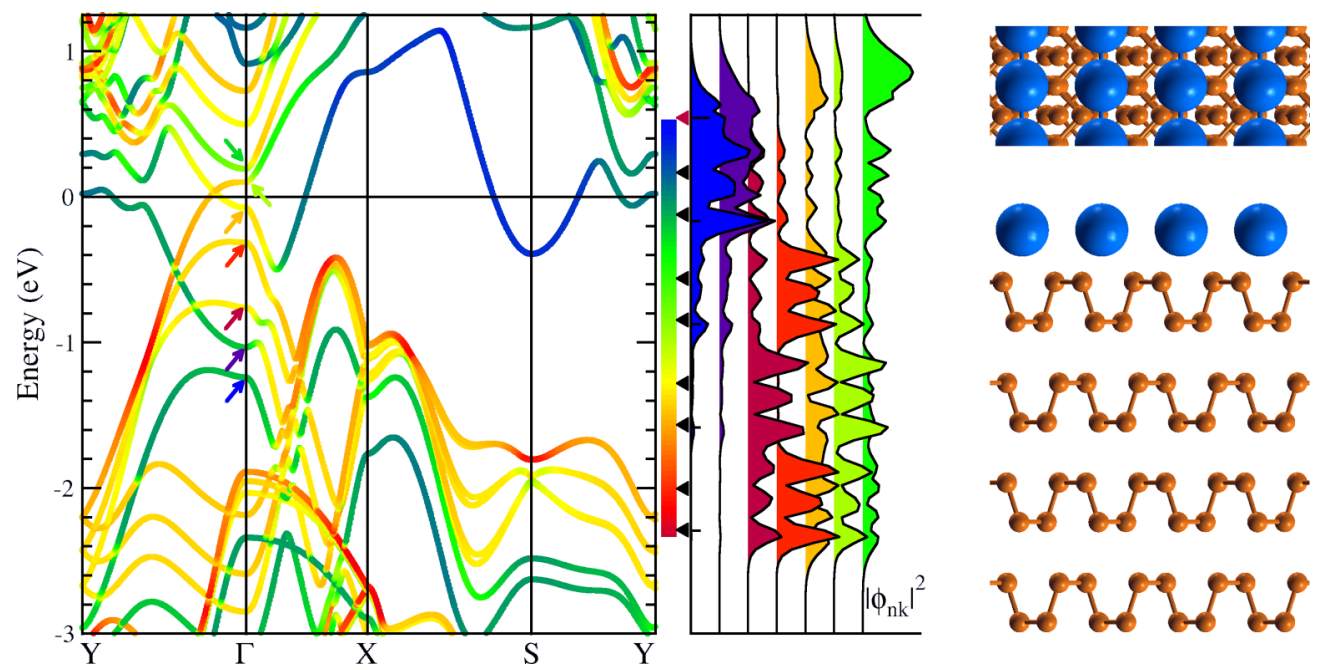

FIG. 7. Left: Band structure of a Cs-doped four-layer phosphorene in the original BP-like geometry. Colors indicate the center of mass of the states according to the color scale in the middle (where black triangles give the position of $\mathrm{P}$ atoms and a red triangle gives that of Cs). Middle: The $x y$ integral of $\left|\phi_{n \mathbf{k}}\right|^{2}$ for selected states (shown by arrows in the band plot). Right: The crystal structure of this system. 
[1] J. Kim, S. S. Baik, S. H. Ryu, Y. Sohn, S. Park, B.-G. Park, J. Denlinger, Y. Yi, H. J. Choi, and K. S. Kim, Observation of tunable band gap and anisotropic dirac semimetal state in black phosphorus, Science 349, 723 (2015).

[2] A. Sanna et al., First-principles and angle-resolved photoemission study of lithium doped metallic black phosphorous, 2D Mater. 3, 025031 (2016).

[3] R. Zhang, J. Waters, A. K. Geim, and I. V. Grigorieva, Intercalant-independent transition temperature in superconducting black phosphorus, Nat. Commun. 8, 15036 (2017).

[4] H. Liu et al., Phosphorene: An unexplored 2D semiconductor with a high hole mobility, ACS Nano 8, 4033 (2014).

[5] S. P. Koenig, R. A. Doganov, H. Schmidt, A. H. Castro Neto, and B. Özyilmaz, Electric field effect in ultrathin black phosphorus, Appl. Phys. Lett. 104, 103106 (2014).

[6] L. Li et al., Black phosphorus field-effect transistors, Nat. Nanotechnol. 9, 372 (2014).

[7] V. Tayari et al., Two-dimensional magnetotransport in a black phosphorus naked quantum well, Nat. Commun. 6, 7702 (2015).

[8] X. Chen et al., High-quality sandwiched black phosphorus heterostructure and its quantum oscillations, Nat. Commun. 6, 7315 (2015)

[9] V. Tayari, N. Hemsworth, O. Cyr-Choinière, W. Dickerson, G. Gervais, and T. Szkopek, Dual-Gate Velocity-Modulated Transistor Based on Black Phosphorus, Phys. Rev. Appl. 5, 064004 (2016).

[10] Q. Guo et al., Black phosphorus mid-infrared photodetectors with high gain, Nano Lett. 16, 4648 (2016).

[11] Y. Saito and Y. Iwasa, Ambipolar insulator-to-metal transition in black phosphorus by ionic-liquid gating, ACS Nano 9, 3192 (2015).

[12] Y. Liu et al., Gate-tunable giant stark effect in few-layer black phosphorus, Nano Lett. 17, 1970 (2017).

[13] C. Han et al., Surface functionalization of black phosphorus via potassium toward high-performance complementary devices, Nano Lett. 17, 4122 (2017).

[14] C. Q. Han et al., Electronic structure of black phosphorus studied by angle-resolved photoemission spectroscopy, Phys. Rev. B 90, 085101 (2014).

[15] E. Golias, M. Krivenkov, and J. Sánchez-Barriga, Disentangling bulk from surface contributions in the electronic structure of black phosphorus, Phys. Rev. B 93, 075207 (2016).

[16] E. Kneedler, D. Skelton, K. E. Smith, and S. D. Kevan, SurfaceState-Surface-Resonance Transition on Ta(011), Phys. Rev. Lett. 64, 3151 (1990).

[17] Q. Liu, X. Zhang, L. B. Abdalla, A. Fazzio, and A. Zunger, Switching a normal insulator into a topological insulator via electric field with application to phosphorene, Nano Lett. 15, 1222 (2015).
[18] S. Yuan, E. van Veen, M. I. Katsnelson, and R. Roldán, Quantum Hall effect and semiconductor-to-semimetal transition in biased black phosphorus, Phys. Rev. B 93, 245433 (2016).

[19] S. S. Baik, K. S. Kim, Y. Yi, and H. J. Choi, Emergence of twodimensional massless Dirac fermions, chiral pseudospins, and Berry's phase in potassium doped few-layer black phosphorus, Nano Lett. 15, 7788 (2015).

[20] B. Ghosh, B. Singh, R. Prasad, and A. Agarwal, Electric-field tunable Dirac semimetal state in phosphorene thin films, Phys. Rev. B 94, 205426 (2016).

[21] J. Kim, S. S. Baik, S. W. Jung, Y. Sohn, S. H. Ryu, H. J. Choi, B.-J. Yang, and K. S. Kim, Two-Dimensional Dirac Fermions Protected by Space-Time Inversion Symmetry in Black Phosphorus, Phys. Rev. Lett. 119, 226801 (2017).

[22] N. Ehlen, B. V. Senkovskiy, A. V. Fedorov, A. Perucchi, P. Di Pietro, A. Sanna, G. Profeta, L. Petaccia, and A. Grüneis, Evolution of electronic structure of few-layer phosphorene from angle-resolved photoemission spectroscopy of black phosphorous, Phys. Rev. B 94, 245410 (2016).

[23] L. Petaccia et al., BaD ElPh: A 4 m normal-incidence monochromator beamline at Elettra, Nucl. Instrum. Methods Phys. Res., Sect. A 606, 780 (2009).

[24] X. Ling et al., Anisotropic electron-photon and electron-phonon interactions in black phosphorus, Nano Lett. 16, 2260 (2016).

[25] B. Kiraly, N. Hauptmann, A. N. Rudenko, M. I. Katsnelson, and A. A. Khajetoorians, Probing single vacancies in black phosphorus at the atomic level, Nano Lett. 17, 3607 (2017).

[26] See Supplemental Material at http://link.aps.org/supplemental/ 10.1103/PhysRevB.97.045143 for animations of the glide mirror symmetry operations for monolayer and bilayer phosphorene. The animation for the bilayer case contains arrows indicating the out-of-plane hopping matrix element $s 1$.

[27] K. Dolui and S. Y. Quek, Quantum-confinement and structural anisotropy result in electrically-tunable Dirac cone in few-layer black phosphorous, Sci. Rep. 5, 11699 (2015).

[28] A. N. Rudenko, S. Yuan, and M. I. Katsnelson, Toward a realistic description of multilayer black phosphorus: From $G W$ approximation to large-scale tight-binding simulations, Phys. Rev. B 92, 085419 (2015).

[29] M. S. Dresselhaus and G. Dresselhaus, Intercalation compounds of graphite, Adv. Phys. 51, 1 (2002).

[30] A. V. Fedorov et al., Observation of a universal donor-dependent vibrational mode in graphene, Nat. Commun. 5, 4257 (2014).

[31] N. I. Verbitskiy et al., Environmental control of electron-phonon coupling in barium doped graphene, 2D Mater. 3, 045003 (2016).

[32] Y. Harada et al., Giant terahertz-wave absorption by monolayer graphene in a total internal reflection geometry, ACS Photonics 4, 121 (2017). 